\title{
A Case of Coil Embolization with Crossing Case Report Y-Configuration Stenting for a Recurrent IC-PC Aneurysm after Clipping
}

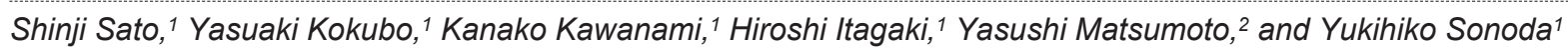

Objective: We report a case in which coil embolization using crossing Y-configuration stenting was effective for an internal carotid-posterior communicating artery (IC-PC) aneurysm with repeated recurrence after clipping.

Case Presentation: The patient was a 57-year-old woman. Nine months after undergoing clipping for a ruptured right IC-PC aneurysm at 55 years of age, she developed a second subarachnoid hemorrhage (SAH) due to recurrence of the aneurysm and underwent clipping at the same site. A third SAH due to rupture of the left IC-PC aneurysm developed 1.5 years after the second clipping. Simultaneously, recurrence of a right IC-PC aneurysm was noted and she was referred to our department. The recurrent right IC-PC aneurysm was considered to have originated from the distal to the initial neck. It was $7 \mathrm{~mm}$ in size and had an irregularly shaped wide neck. As it was assumed that there would be marked adhesion due to repeated surgery, we decided to treat the aneurysm by coil embolization instead of direct surgery. Although the aneurysm neck partially involved the posterior communicating artery (Pcom), tight packing with a minimal residual neck was required. Therefore, crossing Y-configuration stenting was deployed on the internal carotid artery and Pcom using two Neuroform Atlas stents, and coil embolization was performed by the jail technique. The recurrent aneurysm was obliterated. There were no deficits or thrombotic complications after surgery. On DSA follow-up, no compaction or recurrence was observed, and the Pcom was well visualized one year later.

Conclusion: Coil embolization by crossing Y-configuration stenting is a viable treatment option for a recurrent IC-PC wide neck aneurysm.

Keywords $>$ crossing Y-configuration stenting, coil embolization, recurrent aneurysm, internal carotid-posterior communicating artery aneurysm

\section{Introduction}

Although the 10-year incidence of recurrence of intracranial aneurysms at the same site after clipping is $<1 \%$, regardless of rupture, ${ }^{1,2)}$ additional treatment for recurrent aneurysms is often difficult. ${ }^{3,4)}$ The therapeutic strategy, including open surgical and endovascular repair, must be evaluated for each

${ }^{1}$ Department of Neurosurgery, Yamagata University Faculty of Medicine, Yamagata, Yamagata, Japan

${ }^{2}$ Department of Neuroendovascular Therapy, Kohnan Hospital, Sendai, Miyagi, Japan

Received: April 1, 2021; Accepted: July 19, 2021

Corresponding author: Shinji Sato. Department of Neurosurgery, Yamagata University Faculty of Medicine, 2-2-2, Iidanishi, Yamagata, Yamagata 990-9585, Japan

Email: shinjisato.yamagata@gmail.com

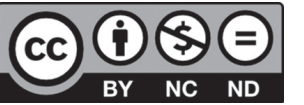

This work is licensed under a Creative Commons Attribution-NonCommercialNoDerivatives International License.

(C)2021 The Japanese Society for Neuroendovascular Therapy patient. Y-configuration stenting (Y-stent-assisted coiling [Y-SAC] $)^{5)}$ using two stents is an adjunctive technique for coil embolization, and there have been many reports on the application of this technique to basilar tip aneurysms, anterior communicating artery (Acom) aneurysms, and middle cerebral artery (MCA) aneurysms. ${ }^{6,7)}$ In contrast, reports on Y-SAC for internal carotid-posterior communicating artery (IC-PC) aneurysms are limited because the placement of one stent in the internal carotid artery (ICA) is often sufficient and Y-SAC is rarely needed. ${ }^{8)}$ We herein report a case in which crossing Y-SAC was effective for treating a recurrent IC-PC aneurysm that repeatedly caused subarachnoid hemorrhage (SAH), even after clipping. This report was approved by the ethics committee of Yamagata University Faculty of Medicine.

\section{Case Presentation}

Patient: A 57-year-old woman.

Complaint: Nothing in particular (treatment requested). 

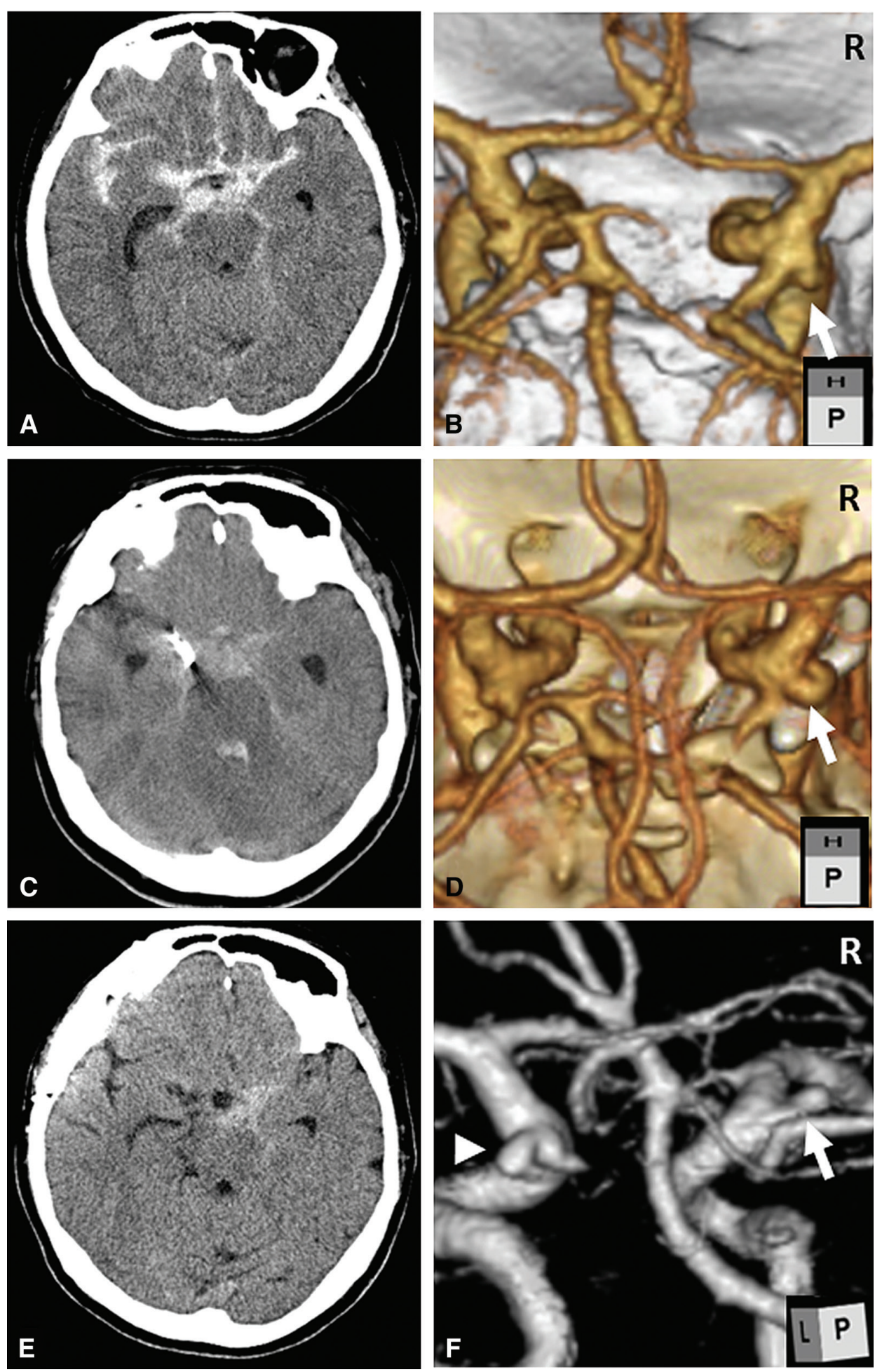

Fig. 1 (A) CT at the first admission showing diffuse and thick SAH in the basal cistern. (B) CTA at the first admission showing the right IC-PC aneurysm (white arrow). (C) CT at the second admission showing diffuse and thin $\mathrm{SAH}$ in the basal cistern. (D) CTA at the second admission showing the recurrent right IC-PC aneurysm (white arrow). (E) CT at the third admission showing the $\mathrm{SAH}$ localized to the left side of the basal cistern. (F) CTA at the third admission showing the de novo left IC-PC aneurysm (white arrowhead) and rerecurrent right IC-PC aneurysm (white arrow). IC-PC: internal carotid-posterior communicating artery; $\mathrm{SAH}$ : subarachnoid hemorrhage
Medical history: Three episodes of SAH.

Family history: Not contributory.

Present illness: The patient had her first episode of SAH at 55 years of age, and under a diagnosis of ruptured right IC-PC aneurysm (Hunt \& Kosnik [H\&K] grade II), she was treated by right frontotemporal craniotomy and clipping at the previous hospital. After surgery, she was discharged to home with a modified Rankin scale (mRS) 1. $\mathrm{SAH}$ recurred 1.5 years later and she was diagnosed with recurrence of the right IC-PC aneurysm (H\&K grade IV). 

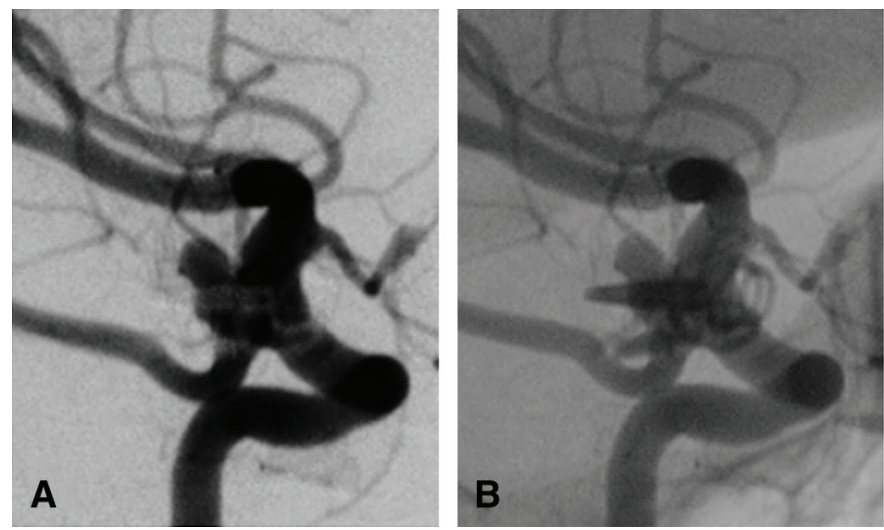

Fig. 2 (A-C) Lateral view on preoperative DSA, DA, and 3D-RA of the right ICA, demonstrating a right IC-PC aneurysm that was irregularly shaped and had a wide neck. (D) Anteroposterior view on left vertebral angiography demonstrating anterograde visualization of the

She underwent clipping and lumbo-peritoneal (LP) shunting at the same hospital, and was discharged to home with an mRS 1. After 2 years, recurrence developed again. It was diagnosed as a ruptured left IC-PC aneurysm (H\&K grade II), and the patient underwent left frontotemporal craniotomy and clipping at the same hospital. On the postoperative examination, recurrence of the right IC-PC aneurysm was detected and the patient was referred to our department for additional treatment.

Condition at the initial examination: The Japan Coma Scale score was 1 and no clear limb paralysis was noted; however, there was a loss of sense of smell and impairment of recent memory, and the mRS score was 1 .

Imaging findings: $\mathrm{CT}$ at the first episode (Fig. 1A and 1B), at recurrence (Fig. 1C and 1D), and at re-recurrence (Fig. 1E and 1F), and images at the time of admission to our department (Fig. 2) are presented. On DSA, the recurrent aneurysm was a wide-necked and irregularly shaped lesion of $7 \mathrm{~mm}$ in maximum dimension that was located postero-laterally to the two clips, mounted on the right posterior communicating artery (Pcom), and extended from the ICA to the Pcom (Fig. 2A-2C). The diameter of the ICA was $4 \mathrm{~mm}$, and the diameter of the Pcom was $3.5 \mathrm{~mm}$ at the origin and $2 \mathrm{~mm}$ at the distal part. Left vertebral angiography clearly visualized the vessels distal to P1 of the right posterior cerebral artery (Fig. 2D).

Course of treatment: The aneurysm recurred repeatedly at the right IC-PC bifurcation, and coil embolization with crossing Y-SAC was selected to tightly embolize the lesion, including the aneurysmal neck. Dual antiplatelet therapy with $100 \mathrm{mg}$ of aspirin and $75 \mathrm{mg}$ of clopidogrel was started 10 days before surgery, and the platelet function was assessed using VerifyNow (Accumetrics, San Diego,
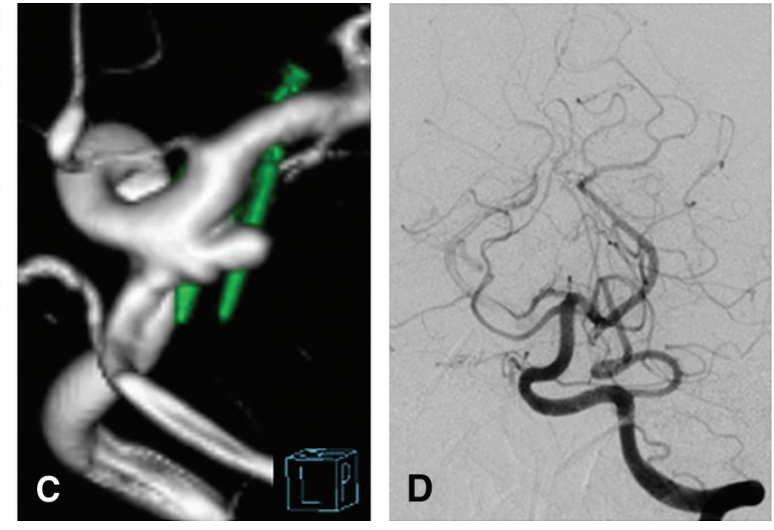

right posterior cerebral artery (positive P1). 3D-RA: 3D rotational angiography; DA: digital angiography; IC-PC: internal carotidposterior communicating artery

CA, USA) 2 days before surgery. A 6-Fr long sheath was placed under general anesthesia, and with systemic heparinization, the activated clotting time was prolonged to 300 seconds. A 6-Fr FUBUKI (Asahi Intecc, Aichi, Japan) was placed at a high position in the cervical segment of the right ICA. To perform coil embolization by the jail technique, a ReSTAR (Medico's Hirata, Osaka, Japan) was placed in the aneurysm. Next, an Excelsior XT-17 (Stryker, Kalamazoo, MI, USA) was guided distally to the M1 of the right MCA. A $4.5 \mathrm{~mm} \times 21 \mathrm{~mm}$ Neuroform Atlas stent (Stryker) was deployed from the proximal side of the right M1 to $\mathrm{C} 2$ of the ICA, across the aneurysm, and the XT-17 was removed. Then, an Excelsior SL-10 (Stryker) was guided to the Pcom by the transcell technique and the SL-10 was placed in the P1 of the right posterior cerebral artery. A $4.0 \mathrm{~mm} \times 21 \mathrm{~mm}$ Neuroform Atlas stent was deployed from the Pcom to $\mathrm{C} 3$ of the ICA and crossing Y-SAC was performed (Fig. 3A). After sufficient framing in the neck of the aneurysm using $6 \mathrm{~mm} \times 10 \mathrm{~cm}$ Target $360 \mathrm{XL}$ soft coils (Stryker) (Fig. 3B), embolization was carried out using a total of 11 coils of $45 \mathrm{~cm}$ in length. At the end of surgery, the patient's condition was classified as class I according to the Raymond Roy Occlusion Classification ${ }^{9)}$ (Fig. 3C).

Postoperative course: No new neurological deficits or thrombotic complications were noted after the procedure (Fig. 4A). Cerebral angiography performed 6 and 12 months after surgery (Fig. 4D and 4E) demonstrated no coil compaction, recurrence of the aneurysm, or thrombotic complication. In the 3 years since the last procedure, the patient has been followed with annual MRI and has remained recurrence free (Fig. 4F). The patient continues to be still followed up on an outpatient basis. 


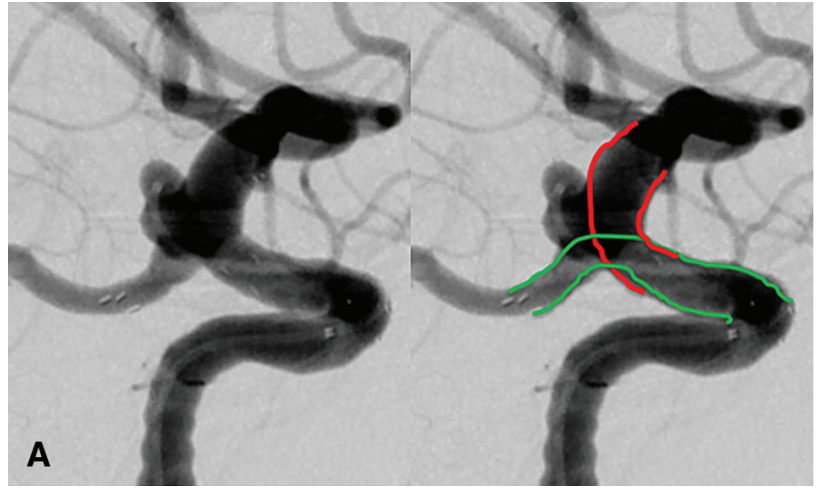

Fig. 3 (A) Intraoperative right carotid angiography in a working projection for stenting. Dual stents were deployed in a Y-configuration. The first stent is shown by the red line and the second stent is shown by the green line. (B) Intraoperative right carotid angiography in a
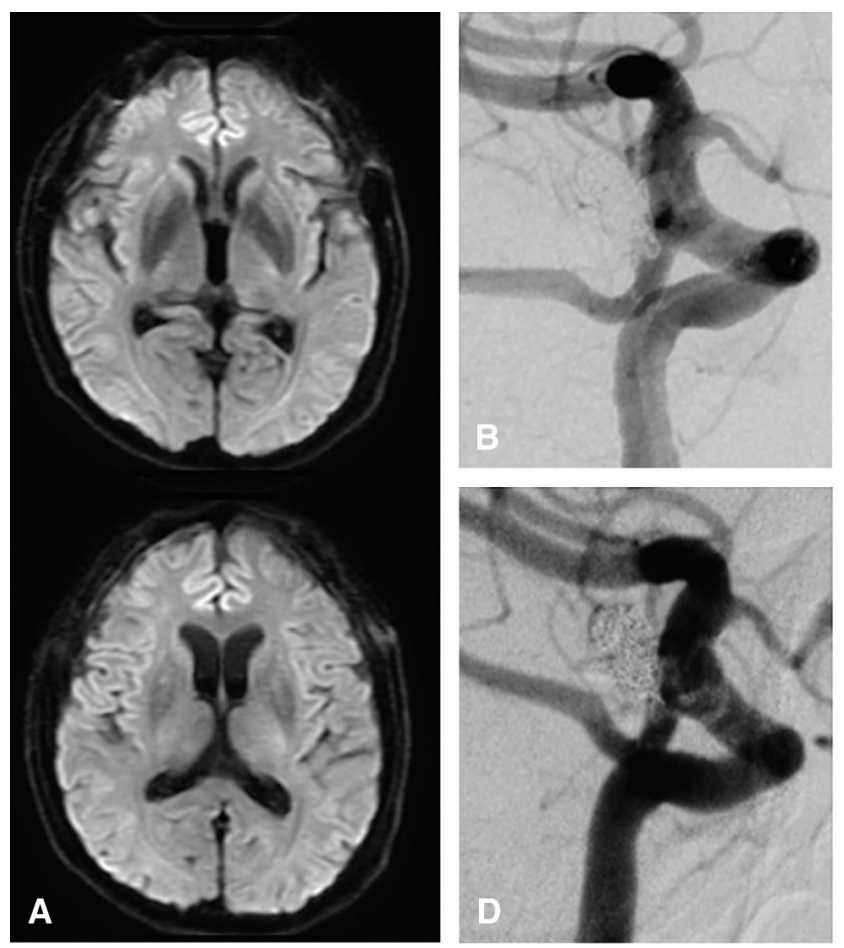

Fig. 4 (A) Diffusion-weighted imaging on the first day after surgery revealed no infarction. (B and $\mathbf{C}$ ) Right internal carotid DSA and DA obtained immediately after coiling. (D and $\mathbf{E}$ ) Follow-up right internal carotid DSA and DA obtained 12 months after coiling, demonstrating

\section{Discussion}

The 10-year incidence of recurrence of aneurysms after craniotomy and clipping is $<1 \%$, regardless of rupture. ${ }^{1-3)}$ Regarding the recurrence of aneurysms after clipping, David et al. evaluated 160 aneurysms in 102 patients and reported that recurrence was observed in two $(1.5 \%)$ of 147 lesions (91.8\%) that were completely occluded, with an annual recurrence rate of $0.52 \% .^{10)}$ In addition, among
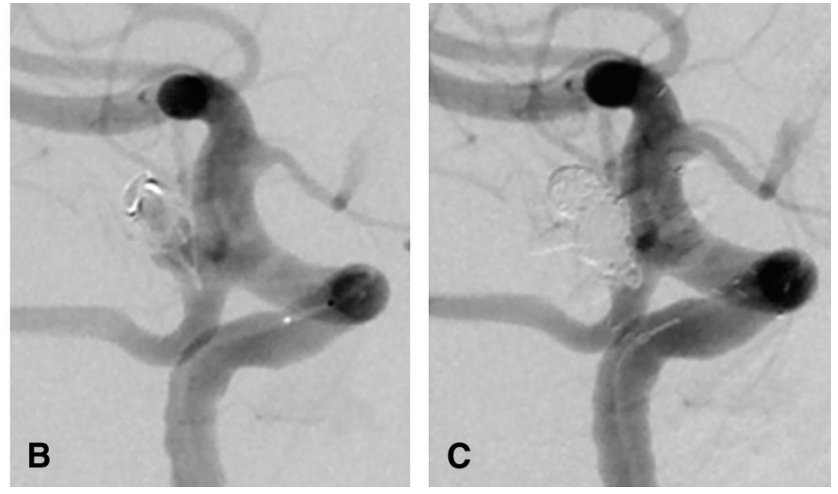

working projection for coiling. The first coil covered the aneurysmal sac well, including the aneurysmal neck. (C) Right internal carotid angiography obtained immediately after coiling, demonstrating obliteration of the aneurysm.
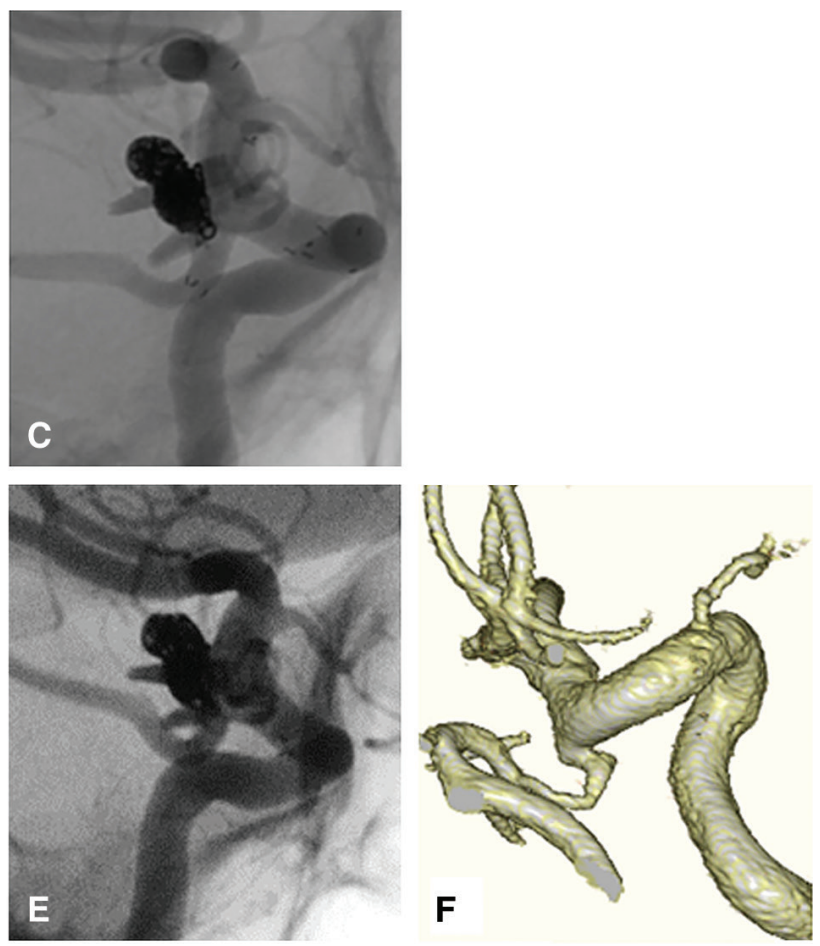

obliteration of the aneurysm. (F) Follow-up right internal carotid high-resolution MRA 3 years after coiling, demonstrating obliteration of the aneurysm. DA: digital angiography

eight aneurysms that exhibited dog-ear remnants, one increased in size, one ruptured, and the annual bleeding rate was $1.9 \%$ when there were residual aneurysms. Treatment by repeat craniotomy for recurrent aneurysms after clipping is often difficult due to the presence of adhesion and clips around the aneurysm, ${ }^{3,4)}$ and Drake et al. reported that intraoperative rupture was likely to occur in patients who receive repeat craniotomy after clipping, that the rate of serious complications was $7.0 \%$, and that the 
mortality rate was $5.2 \% .{ }^{11)}$ If coil embolization is selected for re-treatment after clipping, there are also reports describing aneurysm neck formation due to the clip and noting that complete occlusion is frequently achieved due to the formation of a narrow neck. ${ }^{12,13)}$

In the present case, the right IC-PC aneurysm recurred repeatedly and tight embolization including the aneurysm neck, which was considered to be the site of re-enlargement, was necessary. We also evaluated clipping by repeat craniotomy, but as the patient previously underwent right frontotemporal craniotomy twice, marked adhesion was expected. Furthermore, as the aneurysm originated from the origin of the Pcom behind the two clips, the visibility of the surgical field was expected to be poor (Fig. 2). We selected endovascular treatment based on these factors.

However, the aneurysm had a wide neck and stentassisted coil embolization was necessary. Embolization of the entire aneurysm by deploying the stent into the ICA and sacrificing the Pcom was considered to be the most radical approach. Vertebral angiography visualized the right posterior cerebral artery (PCA) via the right $\mathrm{P} 1$. The Pcom was large in diameter, with a diameter of $3.5 \mathrm{~mm}$ at the origin and $2 \mathrm{~mm}$ at the distal part, and occlusion of the Pcom was considered to involve a risk of infarction of the tuberothalamic artery, which was the penetrating branch of the Pcom. ${ }^{14)}$ Although the lesion was a re-recurring aneurysm, it was unruptured at the time of treatment planning, and we selected intra-aneurysmal embolization by Y-SAC to preserve the Pcom in consideration of the possibility of cerebral infarction due to Pcom occlusion.

Y-SAC is an adjunctive technique involving the placement of two stents in a Y-shaped pattern. The technique was first reported in 2004 by Chow et al.5) Thereafter, there have been many reports on its application to terminal-type aneurysms ${ }^{6,7)}$ and sporadic reports of its application to the IC bifurcation. In contrast, there have been few reports on its application in the treatment of IC-PC aneurysms. ${ }^{8,15-18)}$ According to a meta-analysis of 27 studies, including 750 aneurysms in 744 patients reported by Cagnazzo et al., the complete occlusion rate by Y-SAC was $95.4 \%$, recanalization rate was $3 \%$, the treatment-related complication rate was $8.9 \%$, the morbidity rate was $2.4 \%$, and the mortality rate was $1.1 \%{ }^{6}$ ) These rate differed among the stents used for Y-SAC; the complete occlusion rate was $96 \%$ and the treatment-related complication rate was $6.5 \%$ with the Enterprise (Codman \& Shurtleff, Raynham, MA, USA), the complete occlusion rate was $92 \%$ and the complication rates was $11 \%$ with the
LVIS or LVIS Jr (MicroVention, Tustin, CA, USA), and the complication rates were $94 \%$ and $14 \%$, respectively, with the Neuroform (Stryker). Although a higher complication rate was reported with the Neuroform, this was considered to be due to the use of the Neuroform EZ (Stryker) of a former generation in most patients. In the case of the Neuroform Atlas with a lower metal content, the complete occlusion rate was $93.3 \%$ and the procedurerelated complication rate was low $(6.7 \%)$, with a morbidity rate of $3.3 \%$ according to a study by Aydin et al. that included 30 patients. ${ }^{19)}$

There are 2 Y-SAC procedures: crossing Y-SAC, in which two stents are intersected by placing one stent through the mesh of the other; and kissing Y-SAC, in which two stents are simultaneously deployed in parallel. According to a meta-analysis by Cagnazzo et al., crossing Y-SAC and kissing Y-SAC were performed in 92\% and $8 \%$ of patients, respectively. ${ }^{6)}$ Sato et al. evaluated 38 patients who underwent Y-SAC and reported that crossing Y-SAC and kissing Y-SAC were performed in 19 patients each and that there was no difference in the patient characteristics but that kissing Y-SAC was more often selected for larger wide-necked aneurysms and lesions at the basilar artery (BA) bifurcation in the fetaltype Pcom and at the ICA bifurcation, whereas crossing Y-SAC was more often selected for aneurysms in distal parts of the Acom or MCA. ${ }^{8)}$

The number of microcatheters necessary for treatment is three in kissing Y-SAC (one catheter for intra-aneurysmal embolization and two catheters for simultaneous deployment of two stents), and large-diameter guiding catheters are needed. However, crossing Y-SAC can be performed with two catheters, namely, one for intra-aneurysmal embolization and one for stent deployment. The complication rate is reportedly $8.4 \%$ by crossing Y-SAC and $12.7 \%$ by kissing Y-SAC. ${ }^{6)}$ Recently, T-configuration stenting (T-stent-assisted coiling [T-SAC]), in which two stents are placed in a T-shaped pattern to avoid overlap, was reported to be useful for reducing the risk of thrombotic complications, which is a disadvantage of Y-SAC. ${ }^{20)}$ However, it is technically difficult because the proximal end of the stent placed in the Pcom must be positioned without a gap, with the stent placed in the ICA. According to a report by Aydin et al., thrombotic complications due to occlusion of the Pcom occurred in three of eight patients treated by T-SAC for IC-PC aneurysms and T-SAC is not necessarily considered to be associated with a lower risk of complications than Y-SAC. ${ }^{20)}$ 
Sato $S$, et al.

In the present case, crossing Y-SAC using two Neuroform Atlas stents was selected. We selected Neuroform Atlas for the ICA because the position of the proximal end could be controlled relatively easily and to avoid thrombotic complications due to poor stent deployment in the Pcom. The first stent to be placed in the ICA was deployed from the proximal part of the M1 and was not advanced beyond the $\mathrm{C} 2$ of the ICA, avoiding its deployment in the siphon of the ICA. This facilitated securing of the true lumen of the stent in the ICA with the microcatheter for the second stent and made selection of the Pcom and catheter navigation easier. As the first stent, a short stent $(4.5 \mathrm{~mm} \times 21 \mathrm{~mm})$ was selected because the ICA diameter at the aneurysm neck was $4.5 \mathrm{~mm}$ and as this length could sufficiently cover the neck and make it easy to control the position of the proximal end. Furthermore, as the diameter of the Pcom was $2.0 \mathrm{~mm}$ at the distal part and $3.5 \mathrm{~mm}$ at the origin, and the diameter of the $\mathrm{C} 3$ of the ICA was $4.0 \mathrm{~mm}$, a shorter stent $(4.0 \mathrm{~mm} \times 21 \mathrm{~mm})$ with the same diameter as the ICA was selected as the second stent to prevent migration of the proximal end.

Reports about Y-SAC for IC-PC aneurysms are limited, and a total of 12 cases, including four treated by crossing Y-SAC ${ }^{15-18)}$ and eight treated by kissing Y-SAC, ${ }^{8)}$ were found in our review of the relevant literature. The detailed content of treatment was only described in two cases treated by crossing Y-SAC. ${ }^{15,18)} \mathrm{Kim}$ et al. reported that they performed crossing Y-SAC using two Enterprise stents without postoperative complications or recurrence during a 3-month follow-up period, ${ }^{15)}$ and Raper et al. reported that they performed the procedure using two Neuroform Atlas stents without postoperative complications or recurrence during a 5-month follow-up period. ${ }^{18)}$ There have been very few reports about Y-SAC, probably because patients who require Y-SAC for treatment are rare and because the risk of thrombotic complications increases if the Pcom is thin unless the stents are deployed sufficiently. In our case, the right P1 could be delineated anterogradely by vertebral angiography. The diameter of the Pcom, although not a fetal type, was large $(3.5 \mathrm{~mm}$ at the origin and $2.0 \mathrm{~mm}$ at the distal part) and was sufficient to enable stent deployment in the Pcom. The package insert of the Neuroform Atlas recommends that it be used in vessels with a diameter of at least $2.0 \mathrm{~mm}$. However, Endo et al. reported negative P1 visualization, a Pcom diameter of $\geq 1.8 \mathrm{~mm} \pm 0.4 \mathrm{~mm}$, and a Pcom/P1 ratio of $\geq 1.6 \pm 0.2$ as risk factors for cerebral infarction in the tuberothalamic artery territory due to sacrifice of the Pcom. ${ }^{14)}$ Therefore, in cases involving patients who cannot tolerate sacrifice of the Pcom, including those with a fetal-type Pcom, Y-SAC may be an option with a low risk of thrombotic complications on the condition that the diameter is sufficient for stenting.

\section{Conclusion}

We performed coil embolization with crossing Y-SAC for the treatment of a patient with a recurrent IC-PC aneurysm that repeatedly enlarged after clipping and obtained a favorable postoperative course. Crossing Y-SAC is considered a promising option for the treatment of patients with IC-PC aneurysms in whom the Pcom cannot be sacrificed, when the diameter of the Pcom is sufficient for stenting.

\section{Acknowledgment}

The presentation of this paper was recommended by the chairperson of the 36th Academic Conference. The authors express their sincere gratitude.

\section{Disclosure Statement}

Yasushi Matsumoto received patent royalties from Sumitomo Bakelite Co., Ltd. and rewards for lectures, etc., from Kaneka Medix Corp., Medico's Hirata Inc., and Fuji Systems Corporation.

\section{References}

1) Tsutsumi K, Ueki K, Usui M, et al. Risk of recurrent subarachnoid hemorrhage after complete obliteration of cerebral aneurysms. Stroke 1998; 29: 2511-2513.

2) Tsutsumi K, Ueki K, Usui M, et al. Risk of subarachnoid hemorrhage after surgical treatment of unruptured cerebral aneurysms. Stroke 1999; 30: 1181-1184.

3) Shimizu H, Yanagisawa $T$, Endo $H$, et al. Surgery for aneurysms that are residual or recurrent after open or endovascular surgery. Surg Cereb Stroke (Jpn) 2015; 43: 212-217.

4) Kajimoto R, Shimada K, Kano T, et al. Effect of endovascular coil embolization for recurrent cerebral aneurysms following surgical clipping. Jpn J Stroke 2018; 40: 249-254. (in Japanese)

5) Chow MM, Woo HH, Masaryk TJ, et al. A novel endovascular treatment of a wide-necked basilar apex aneurysm by using a Y-configuration, double-stent technique. AJNR Am J Neuroradiol 2004; 25: 509-512. 
6) Cagnazzo F, Limbucci N, Nappini S, et al. Y-stent-assisted coiling of wide-neck bifurcation intracranial aneurysms: a meta-analysis. AJNR Am J Neuroradiol 2019; 40: 122-128.

7) Granja MF, Cortez GM, Aguilar-Salinas P, et al. Stentassisted coiling of cerebral aneurysms using the Y-stenting technique: a systematic review and meta-analysis. $J$ Neurointerv Surg 2019; 11: 683-689.

8) Sato K, Matsumoto Y, Kanoke A, et al. Y-Configuration stenting for coil embolization of complex intracranial aneurysms: distinguishing between use of crossing-Y and kissing-Y. World Neurosurg 2021; 146: e1054-e1062.

9) Mascitelli JR, Moyle H, Oermann EK, et al. An update to the Raymond-Roy occlusion classification of intracranial aneurysms treated with coil embolization. J Neurointerv Surg 2015; 7: 496-502.

10) David CA, Vishteh AG, Spetzler RF, et al. Late angiographic follow-up review of surgically treated aneurysms. J Neurosurg 1999; 91: 396-401.

11) Drake CG, Friedman AH, Peerless SJ. Failed aneurysm surgery. Reoperation in 115 cases. J Neurosurg 1984; 61: 848-856.

12) Forsting M, Albert FK, Jansen O, et al. Coil placement after clipping: endovascular treatment of incompletely clipped cerebral aneurysms. Report of two cases. J Neurosurg 1996; 85: 966-969.

13) Cekirge HS, Islak C, Firat MM, et al. Endovascular coil embolization of residual or recurrent aneurysms after surgical clipping. Acta Radiol 2000; 41: 111-115.
14) Endo H, Sato K, Kondo R, et al. Tuberothalamic artery infarctions following coil embolization of ruptured posterior communicating artery aneurysms with posterior communicating artery sacrifice. AJNR Am J Neuroradiol 2012; 33: 500-506.

15) Kim TG, Kim SH, Cho KG, et al. Endovascular treatment of internal carotid-posterior communicating artery wide-necked aneurysm using 2 Enterprise stents in Y-configuration. Clin Neurol Neurosurg 2013; 115: 1117-1120.

16) Bang JS, Kim CH, Kwon BJ, et al. The difficulties and risks of Y-stent-assisted coiling: a comparison of first and second stenting procedures. World Neurosurg 2016; 88: 146-153.

17) Castaño $C$, Terceño $M$, Remollo $S$, et al. Endovascular treatment of wide-neck intracranial bifurcation aneurysms with ' $\mathrm{Y}$ '-configuration, double Neuroform ${ }^{\circledR}$ stents-assisted coiling technique: experience in a single center. Interv Neuroradiol 2017; 23: 362-370.

18) Raper DMS, Rutledge WC, Winkler EA, et al. Y-stent technique for treatment of wide-necked posterior communicating artery aneurysm associated with fetal posterior cerebral artery: technical report. World Neurosurg 2020; 133: 173-177.

19) Aydin K, Balci S, Sencer S, et al. Y-stent-assisted coiling with low-profile Neuroform Atlas stents for endovascular treatment of wide-necked complex intracranial bifurcation aneurysms. Neurosurgery 2020; 87: 744-753.

20) Aydin K, Stracke CP, Barburoglu M, et al. Long-term outcomes of wide-necked intracranial bifurcation aneurysms treated with T-stent-assisted coiling. J Neurosurg 2019; 6: $1-10$. 